\title{
Integración de las Etnomatemáticas en el Aula de Matemáticas: posibilidades y limitaciones
}

\section{Integration of Ethnomathematics in Mathematics Classroom: possibilities and limitations}

\author{
María Luisa Oliveras* \\ Hilbert Blanco-Álvarez ${ }^{* *}$
}

\begin{abstract}
Resumen
Este artículo presenta la primera parte de una investigación doctoral sobre la formación de maestros, enfocada desde las Etnomatemáticas. Se analiza la veracidad de dos conjeturas: $\mathrm{C} 1$. Los maestros no reconocen prácticas extraescolares como conocimientos matemáticos; y C2. Esta convicción obstaculiza el uso de prácticas extraescolares en el aula. Partiendo de estas conjeturas, planificamos un curso de formación, desde la Etnomatemática, para maestros de educación primaria en Colombia. Dicho curso se tomó como caso a investigar mediante una metodología cualitativa de estudio de casos. Se han analizado, bajo la teoría filosófica de Wittgenstein, los registros obtenidos de una entrevista grupal y del diario de campo del investigador. Concluimos que las conjeturas no se cumplen, ya que no existe total coherencia entre las concepciones epistemológicas y la acción en el aula. Además, emergieron de la investigación otros elementos que inciden en la conexión entre creencias y prácticas en aula interesantes para tener en cuenta en la formación de profesores.
\end{abstract}

Palabras-clave: Formación de maestros que enseñan matemáticas. Etnoeducación. Currículo. Matemáticas extraescolares. Diversidad cultural.

\begin{abstract}
This paper presents the first part of a doctoral research about teacher's training, focused on Ethnomathematics. It analyzed the veracity of two conjectures: $\mathrm{C} 1$. Teachers do not recognize mathematical extracurricular activities as math, and $\mathrm{C} 2$. This conviction obstructs the use of this knowledge in the classroom. Starting from these conjectures, we planned a training course, on ethnomathematics, for primary school teachers in Colombia. The course was taken as an investigative case through a qualitative methodology for case studies. The records produced from a group interview and researcher's field diary were analyzed under the Wittgenstein's philosophy view. We concluded that the conjectures are not met, since there is no complete consistency between the epistemological concepts and action in the classroom. Also, they emerged from researching other elements which affect the connection between beliefs and classroom practices interesting to reflect on teacher training.
\end{abstract}

Keywords: Math teacher training. Ethnoeducation. Curriculum. Extracurricular mathematics. Cultural diversity.

\footnotetext{
* Doctora en Matemáticas por la Universidad de Granada (UGR). Investigadora y Profesora Titular de Didáctica de la Matemática en Universidad de Granada (UGR). Facultad de Ciencias de la Educación, Granada, España. Dirección Postal: Campus Cartuja s/n 18079, Granada, España. E-mail: oliveras@ugr.es

** Doctorando en Educación por la Universidad de Granada (UGR). Facultad de Ciencias de la Educación y Docente de la Universidad de Nariño, Pasto, Nariño, Colombia. Dirección Postal: Facultad de Ciencias Exactas y Naturales, Universidad de Nariño, Campus Torobajo, Pasto, Nariño, Colombia. E-mail: hilbla@udenar.edu.co
} 


\section{Introducción}

Este artículo presenta resultados parciales de una investigación doctoral en curso ${ }^{1}$. El objetivo general de la investigación es identificar elementos para el diseño de programas de formación de maestros que enseñan matemáticas desde la Etnomatemática. Para la identificación de dichos elementos se plantearon distintas conjeturas, definidas a la luz del modelo teórico MEDIPSA, elaborado por Oliveras (1996), y la experiencia previa acumulada de los autores sobre el conocimiento de que estos maestros no fueron formados bajo una perspectiva sociocultural de la Educación Matemática.

Las primeras conjeturas que se trabajaron, y de las cuales se presentan los resultados en este artículo, son expresadas como: Conjetura 1: Los maestros no reconocen prácticas extraescolares como conocimientos matemáticos, de la que se deriva la Conjetura 2: esta convicción obstaculiza el uso de prácticas extraescolares en el aula de clase.

Estas conjeturas orientaron el diseño y la ejecución de la primera parte de un Curso de Formación de Maestros desde la Etnomatemática (CFME) dirigido a maestros de educación primaria en Tumaco, Colombia. Este CFME es el caso de estudio que analizamos para confirmar o refutar la veracidad de las conjeturas 1 y 2.

\section{Problemática y antecedentes}

El reconocimiento, o el dar estatus de matemáticas, por parte de los maestros, a prácticas extraescolares genera tensión entre el saber escolar y el saber cotidiano, puesto que el primero se considera científico y el segundo no. Dichas tensiones son reconocidas en distintas investigaciones como (BLANCO-ÁLVAREZ, 2011; D’AMBROSIO, 1997; MONTEIRO; MENDES, 2011; JARAMILLO, 2011; KNIJNIK; MEREGALLI, 2012; OLIVERAS, 1996, 2006; VILELA, 2007, entre otros).

Algunas de las características que diferencian las matemáticas escolares de las matemáticas extraescolares son presentadas por Vilela (2007) en el Cuadro 1.

Estas características ilustran las diferencias entre una y otra en términos de los objetivos que persiguen, tipos de respuestas, significados institucionales y personales,

\footnotetext{
1 Investigación doctoral titulada Elementos para la formación de maestros de matemáticas desde una perspectiva Etnomatemática, dirigida por la Dra. María Luisa Oliveras dentro del Grupo de Investigación Etnomatemáticas, Formación de profesores y Didáctica de la Universidad de Granada, España.
} 
procesos algorítmicos, ente otros, donde, históricamente, las características de la matemática escolar han sido privilegiadas, en particular la escritura (KNIJNIK, 2007).

\begin{tabular}{|l|l|}
\hline Características de la matemática escolar & Características de las matemática extraescolares \\
\hline Situaciones generales & Situaciones particulares \\
\hline Énfasis en algoritmos, fórmulas, secuencias & Esfuerzo de resolver problemas \\
\hline $\begin{array}{l}\text { Disociación de los cálculos de las actividades } \\
\text { reales, llegando a respuestas sin sentido }\end{array}$ & Aproximaciones y redondeamientos sensatos \\
\hline Deductiva & Inductiva \\
\hline Solución correcta y superior & Solución adecuada \\
\hline Escrito & Oral \\
\hline Generalización & No generalización \\
\hline Formal & Informal \\
\hline Resultado y número & Resultado y decisión \\
\hline Número puro & Número de cosas reales \\
\hline Resultados únicos & Resultados aproximados \\
\hline Cálculos escritos & $\begin{array}{l}\text { Cálculos mentales: procesos de agrupamiento y } \\
\text { redondeo }\end{array}$ \\
\hline Sin significado para el alumno & Con significados para quien las hace \\
\hline
\end{tabular}

Cuadro 1 - Características de la matemática escolar VS la matemática extraescolar

Por otra parte, existe en la literatura internacional un amplio número de investigadores en Educación Matemática y en Etnomatemática que están de acuerdo en integrar las matemáticas extraescolares en el currículo escolar (CARRAHER; CARRAHER; SCHLIEMANN，2002; SCHLIEMANN，2002; SANTILLÁN; ZACHMAN，2009, entre otros). Sin embargo, no siempre dicha integración obedece a los mismos intereses. Vilela (2006) presenta al menos tres enfoques diferentes mediante los cuales es posible hacer dicha integración: i. Cognitivo; ii. Amplificador; y iii. Político.

El interés cognitivo se refiere a la importancia y la necesidad de integrar las matemáticas extraescolares en el aula de clase, favoreciendo la búsqueda de mayor significado del contenido matemático escolar. Puesto que en la escuela el significado:

\footnotetext{
[...] se pierde porque la resolución de problemas tiene objetivos que difieren de aquellos que nos mueven para resolver problemas fuera del aula, además porque en la clase no estamos preocupados por situaciones particulares sino por reglas generales que tienden a vaciar el significado de las situaciones o porque la profesora no le interesa el esfuerzo de un alumno por resolver el problema sino la aplicación de una formula, de un algoritmo, de una operación predeterminados por el capítulo en que se inserta el problema o por el año escolar en que está el niño (CARRAHER; CARRAHER; SCHLIEMANN, 2002, p. 22).
}

Asimismo, Schliemann (2002, p.104) argumenta que "los mejores resultados se obtienen cuando la experiencia diaria se combina con la experiencia escolar".

El interés amplificador tiene que ver con acrecentar el conocimiento matemático escolar gestionado mediante el currículo, al incorporar al aula los conocimientos de las matemáticas extraescolares y los saberes previos de los estudiantes, y reflexionar con ellos sobre éstos. Knijnik (1996 citada en VILELA, 2006) propone llevar la matemática 
extraescolar al aula a través de comparaciones de procedimientos matemáticos típicos de la escuela con los de la calle y promover una discusión respecto de los límites y alcances de cada método matemático.

El interés político intenta reivindicar la legitimidad de las matemáticas extraescolares, buscando la equidad social y el reconocimiento de éstas en la escuela. Al respecto, Oliveras (1996, p.75) argumenta que:

[...] un diálogo en el que el profesor hable desde sus supuestos etnomatemáticos, generalmente desarrollados en la Universidad, y los alumnos hablen con los suyos, produce conocimiento matemático y puede llevar a los alumnos a afianzar sus raíces socio-culturales, porque sus conocimientos "etno" son legitimizados, reconocidos y valorados, en el proceso educacional.

En este mismo sentido, Jaramillo (2011, p. 17) señala que "cuando los saberes escolares desconocen o deslegitiman otra forma de conocimientos y de saberes, se genera un modo de exclusión social, pues esto conlleva a la deslegitimación de las prácticas sociales que dan sustento a dichos saberes".

Por lo tanto, la necesidad de integrar las matemáticas extraescolares al trabajo en la escuela queda argumentada y también son reconocidas las tensiones que provoca esta integración.

\section{Marco teórico}

Esta investigación se enmarca en el Programa de Etnomatemática planteado por D’Ambrosio (2012) y utiliza el modelo teórico MEDIPSA, desarrollado por Oliveras (1996), como caja de herramientas para realizar el análisis e interpretación de los datos. Dicho modelo está conformado por distintas teorías, que son compatibles entre sí, de disciplinas establecidas como constituyentes de las siete componentes del fenómeno didáctico-matemático: Matemáticas, Epistemología, Didáctica, Investigación Interpretativa, Psicología, Sociología y Antropología. Es un modelo que fue elaborado para fundamentar la investigación sobre formación de profesores en Etnomatemática. En particular, mostramos el uso que se ha hecho de la componente Matemáticas, Epistemología y Psicología en la primera parte de la investigación.

\subsection{Base epistemológica de la Etnomatemática}


La filosofía de las matemáticas que Wittgenstein (1999) desarrolla en su libro Investigaciones Filosóficas, resulta muy útil para la base epistemológica de la Etnomatemática, en tanto que nos permite reconocer distintas racionalidades, visiones del mundo y formas de legitimación del conocimiento. "Wittgenstein (...) establece otra teoría del significado basada en el uso público del lenguaje y en la noción de 'juego lingüístico', lo que indica su cambio hacia una concepción del mundo y del proceso cognoscitivo interactiva o epistemológicamente transaccional" (OLIVERAS, 1996, p. 68), y donde "cada lenguaje es un modo de conocer que ha desarrollado un grupo de seres humanos" (OLIVERAS, 1996, p. 74). En este mismo sentido, Knijnik (2007, p.75) señala que "Wittgenstein ayuda a fundamentar filosóficamente el campo de la Etnomatemática, especialmente en lo que dice respecto a la no existencia de una sola matemática, esa que llamamos "la" Matemática, asociada a la racionalidad moderna".

Estamos de acuerdo con Knijnik (2012), en que los conceptos de Wittgenstein que apoyan la investigación Etnomatemática son: juegos de lenguaje, formas de vida y semejanzas de familia.

Los juegos de lenguaje hacen referencia a los distintos usos que se le dé a una palabra, de los cuales van a depender los diferentes significados que ésta pueda tener en diversos contextos. "El significado de una palabra es su uso en el Lenguaje" (WITTGENSTEIN, 1999, \# 43, p. 17). De este modo, es posible abandonar cualquier esencialismo del lenguaje, dado que las palabras no tendrían significados universales pues dependen del juego de lenguaje del que participen. A este respecto, Wittgenstein $(1999, \# 1$, p. 7) se pregunta: “¿Pero cuál es el significado de la palabra "cinco"?- No se habla aquí en absoluto de tal cosa; sólo de cómo se usa la palabra "cinco"”.

Así pues, concordamos con Knijnik (2006) y Vilela (2007), en considerar "la matemática académica como un conjunto de juegos de lenguaje que no puede pretender ser único y universal. De este modo, es posible hablar de diferentes redes de juegos de lenguaje, por extensión, de diferentes matemáticas” (KNIJNIK; DUARTE, 2010, p. 869, traducción nuestra)

Las matemáticas son, para Wittgenstein (1999, p.123-124):

Una convención $[\ldots]$ si no hubiera ese acuerdo completo, la gente tampoco aprendería la técnica que aprendemos. Sería distinta de la nuestra en mayor o menor medida, incluso hasta llegar a ser irreconocible. [...] Naturalmente, la matemática es, en cierto sentido, una doctrina.

En este mismo sentido, Lizcano (2006, p.196-197) señala que: 
[...] nuestras matemáticas, las que solemos llamar simplemente 'matemáticas', también nacieron allí, en cierto lugar. Un lugar en el que habitaban, y siguen habitando, ciertas gentes con una manera muy especial de vivir y de pensar, con una manera muy especial de medir, razonar y calcular.

Y, más adelante, expone: "Nuestra aritmética, decía Wittgenstein, se sostiene como se sostiene cualquier otra institución social: porque mucha gente cree en ella (LIZCANO, 2006, p. 202).

Ahora, para conocer el significado de una palabra es necesario conocer su uso en determinado contexto, es decir su juego, pero para conocer el juego del que participa es necesario, también, conocer las reglas de dicho juego, su gramática, que nos permite reconocer si una palabra o una oración tienen sentido en dicho juego, su lógica. Pero este contexto está cargado de cultura, de visiones del mundo, de prácticas, de actos no lingüísticos, de valores, entre otros. Este contexto es llamado por Wittgenstein Formas de vida: "La expresión 'juego de lenguaje’ debe poner de relieve aquí que hablar el lenguaje forma parte de una actividad o de una forma de vida" (WITTGENSTEIN, 1999, \# 23, p. 12); además, “imaginar un lenguaje significa imaginar una forma de vida" (WITTGENSTEIN, 1999, \# 19, p. 10).

Es, entonces, en las formas de vida donde habitan los juegos de lenguaje y donde adquieren significados. En palabras de Glock (1998 citado por VILELA, 2007, p. 147):

Los juegos de lenguaje están interrelacionados con el contexto. Un lenguaje se establece colectivamente, pues el significado no es privado, sino social, fruto de convenciones resultantes de antiguos acuerdos comunitarios. Los significados y la comprensión, también ligados al lenguaje, están asociados al sonido, al contexto del que participan, a los modos de comunicación; comprender es una capacidad manifiesta en el uso (Traducción nuestra).

El significado, entonces, de una palabra, depende del juego del que participe en el interior de una forma de vida. También, una palabra podría tomar distintos significados en una misma forma de vida. Por ejemplo en una clase de matemáticas escolares la letra $x$ podría participar en distintos juegos de lenguaje, cada uno con reglas diferentes en donde el significado varía. Así pues, la $x$ podría ser el nombre de un vértice de un triángulo, la $x$ podría ser una incógnita y la $x$ podría ser una variable. Si un estudiante desconoce las reglas de juego en el cual está siendo enunciada la $x$, con toda certeza éste caerá en error. Así mismo, podría ocurrir con palabras que utilizamos fuera del aula de matemáticas, como: función, límite, real, campo, vecindad, imaginario, complejo, integral, entero etc., que tienen significados muy diferentes en formas de vida extraescolares.

A este respecto, Vilela (2007, p.12) llama la atención sobre la diferencia de las reglas de las matemáticas escolares y las reglas de las matemáticas extraescolares, por ejemplo: 
Las reglas de la matemática escolar generalmente son pautadas en una lógica de reglas fijas de la lógica clásica cuya presencia en la matemática de la calle es bastante diferente o alterada, estos es, orientada por otros valores (Traducción nuestra).

Así pues, Vilela (2010, p. 350) argumenta que:

Las prácticas matemáticas usadas en la calle, en las escuelas, en la academia, o por grupos profesionales etc., son un conjunto variado de juegos de lenguaje o diferentes usos de conceptos matemáticos en prácticas diferentes y por lo tanto no constituyen un único edificio de conocimiento llamado matemáticas, sino esquemas teóricos específicos que forman condiciones para el sentido, significado e inteligibilidad en diferentes situaciones, tiempos y lugares en la vida (Traducción nuestra).

Pero si las matemáticas no constituyen un único edificio de conceptos universales, sino por el contrario, son diversos edificios interconectados que tienen lugar en distintas formas de vida ¿cómo podemos reconocer qué es matemático en cada forma de vida? Es decir, ¿cómo podemos señalar que las comunidades afrodescendientes tienen sus propios patrones arbitrarios de medición de longitudes o las comunidades indígenas tienen sus propias formas de localización, entre muchas otras cosas? Wittgenstein nos dota de una herramienta filosófica para esto, las semejanzas de familia, pues, al no existir ya conceptos esencialistas, no es posible hablar de un núcleo común entre los conceptos, a lo sumo podremos hablar de semejanzas, de parecidos. Wittgenstein lo expresa muy bien con el aforisma \# 66 donde intenta explicar que la palabra juego no admite una definición cerrada:

66. Considera, por ejemplo, los procesos que llamamos 'juegos'. Me refiero a juegos
de tablero, juegos de cartas, juegos de pelota, juegos de lucha, etc. ¿Qué hay común
a todos ellos? - No digas: 'Tiene que haber algo común a ellos o no los
llamaríamos 'juegos' - sino mira si hay algo común a todos ellos. - Pues si los
miras no verás por cierto algo que sea común a todos, sino que verás semejanzas,
parentescos y por cierto toda una serie de ellos. Como se ha dicho: ¡no pienses, sino
mira! Mira, por ejemplo, los juegos de tablero con sus variados parentescos. Pasa
ahora a los juegos de cartas: aquí encuentras muchas correspondencias con la
primera clase, pero desaparecen muchos rasgos comunes y se presentan otros. Si
ahora pasamos a los juegos de pelota, continúan manteniéndose varias cosas
comunes pero muchas se pierden. - ¿Son todos ellos 'entretenidos'? Compara el
ajedrez con el tres en raya. ¿O hay siempre un ganar y perder, o una competición
entre los jugadores? Piensa en los solitarios. En los juegos de pelota hay ganar y
perder; pero cuando un niño lanza la pelota a la pared y la recoge de nuevo, ese
rasgo ha desaparecido. Mira qué papel juegan la habilidad y la suerte. Y cuan
distinta es la habilidad en el ajedrez y la habilidad en el tenis. Piensa ahora en los
juegos de corro: Aquí hay el elemento del entretenimiento, ipero cuántos de los
otros rasgos característicos han desaparecido! Y podemos recorrer así los muchos
otros grupos de juegos. Podemos ver cómo los parecidos surgen y desaparecen.
Y el resultado de este examen reza así: Vemos una complicada red de parecidos que
se superponen y entrecruzan. Parecidos a gran escala y de detalle (WITTGENSTEIN
1999, p. 22-23).

Así entonces, concordamos con Knijnik (2012, p. 92) en que mediante la noción de semejanzas de familia es posible argumentar que algunos juegos de lenguaje de formas de vida extraescolares son matemáticas porque identificamos semejanzas entre ellos y algunos 
juegos de lenguaje de las formas de vida académicas. Este puede ser, entonces, un criterio a ser usado para decidir si un juego de lenguaje es matemático o no. Con este criterio también podemos dar respuesta a la crítica que formuló Milroy (citado en FERREIRA, 1997, p. 24): “¿cómo puede alguien que fue escolarizado dentro de la matemática occidental convencional 'ver' cualquier otra forma de matemática que no se parezca a la matemática convencional, que le es familiar?", pues no hay una realidad independiente del punto de vista del observador. Este es un problema al cual el investigador en etnomatemática está forzosamente condenado, puesto que no existe una realidad pura, independiente del punto de vista de nadie, toda observación depende de las condiciones del observador, es inevitable. Y, en nuestro caso, aquellas condiciones iniciales se refieren a estar formados en la matemática difundida por occidente.

Epistemológicamente, las nociones de juegos de lenguaje, formas de vida, $\mathrm{y}$ semejanzas de familia nos permiten reconocer distintas prácticas culturales como matemáticas, cada una de ellas inmersas en la cultura de cada grupo social, con unas reglas propias de organización y legitimación del conocimiento matemático, donde la mayor semejanza que tienen con la matemática académica es su interés por los problemas de la comunidad y la imperativa necesidad de resolverlos eficientemente.

En este sentido, es posible hablar de las matemáticas de los palenqueros, los indígenas, los carpinteros, los matemáticos, los campesinos u otros grupos culturales, cuyas matemáticas tendrán semejanzas entre ellas, más en el qué se centran que en el cómo se realizan sus prácticas.

\subsection{Noción de concepción}

Respecto a la componente psicológica del MEDIPSA, consideramos las concepciones de los maestros como:

[...] una estructura más general, incluyendo creencias, significados, conceptos, proposiciones, reglas, imágenes mentales, preferencias y similares. Aunque puede que la distinción no tenga una gran importancia, en ocasiones será más natural referirse a las concepciones de los profesores sobre las matemáticas como disciplina, que hablar simplemente de las creencias de los profesores sobre las matemáticas (THOMPSON, 1992, p. 130).

[...] Una concepción del profesor sobre la naturaleza de las matemáticas puede verse como creencia, concepto, significado, regla, imagen mental y preferencia, consciente o inconsciente del profesor en relación con las matemáticas. Éstas creencias, conceptos, puntos de vista y preferencias, constituyen los rudimentos de una filosofia de las matemáticas" (THOMPSON, 1992, p.132, traducción nuestra). 
Una epistemología personal de las matemáticas de cada maestro se materializa en unas prácticas educativas que se expresan en un lenguaje personal de los maestros.

\section{Diseño de la investigación y del caso}

\subsection{Metodología de la investigación}

Para estudiar la veracidad de las Conjeturas 1 y 2 se realizó una investigación cualitativa, de carácter interpretativo, siguiendo una aproximación etnográfica. El método de investigación es un estudio de casos. El caso es un Curso de Formación desde la Etnomatemática para maestros en ejercicio, realizado en el municipio de Tumaco, Colombia entre julio y octubre de 2012. Este se describe en detalle más adelante.

\subsubsection{Etapas de la investigación}

La figura 1 presenta las diferentes etapas de la investigación, iniciando con la definición del objetivo general, las conjeturas de la investigación y la metodología, continuando con el diseño del curso, luego su implementación y producción de registros, su análisis y, finalmente, la verificación de las conjeturas inicialmente planteadas.

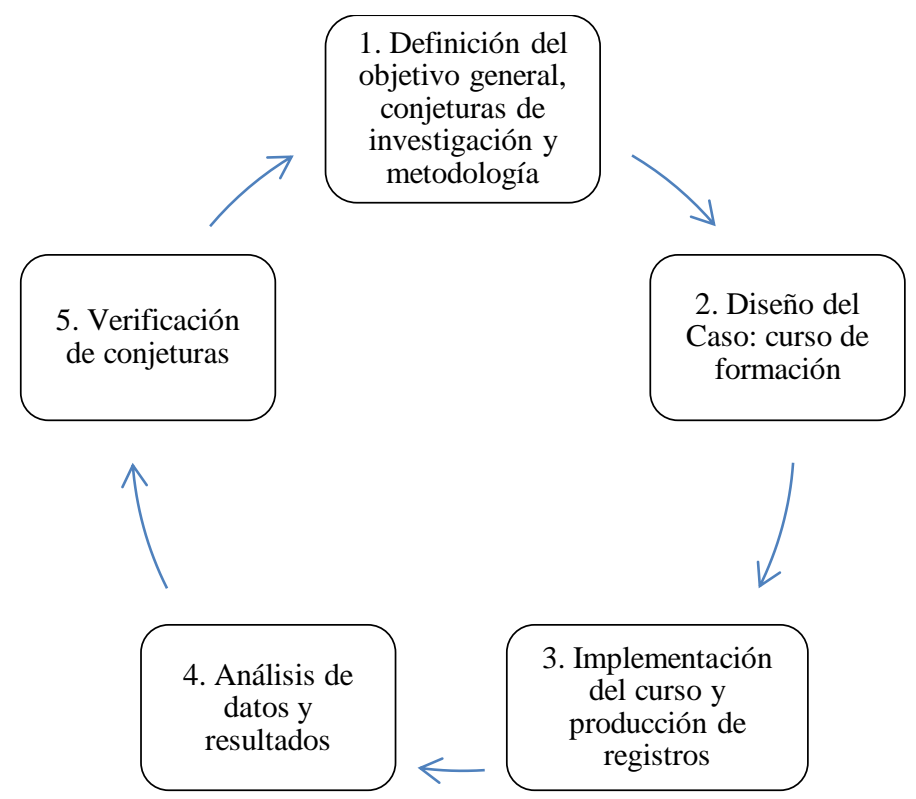

Figura 1 - Etapas de la investigación.

\subsubsection{Rol de los investigadores}


En la investigación, el segundo autor de este artículo jugó un doble rol. Por un lado, el papel de investigador junto al primer autor, quienes diseñaron la investigación, el curso como parte del proceso investigativo y el análisis posterior. Por el otro, el segundo autor asumió el rol de profesor y observador participante, al realizar el desarrollo del curso, teniendo acciones como profesor que, a su vez, analiza, en el rol de investigador. Por tanto la visión del segundo autor es doble y compleja.

\subsubsection{Producción de registros}

En el desarrollo del Curso de formación, analizado en esta parte de la investigación, se hizo uso de la entrevista grupal, puesto que se consideró importante y necesario que los maestros participantes escucharan las opiniones de los demás y, así, generar discusión para que de manera natural fueran emergiendo las posiciones epistémicas de los maestros (COHEN; MANION, 1990). La discusión en la entrevista fue motivada a partir de un instrumento constituido por una lista de diecisiete frases, tomadas de Blanco-Álvarez (2012), que se presenta en la figura 2. La entrevista grupal fue registrada en grabaciones de audio.

i. Las mujeres africanas que tejen figuras como cuadrados, círculos, triángulos etc. en los cestos o en la ropa tienen nociones de geometría.

ii. Son adecuados los patrones de medida como el pie y el palmo que muchos albañiles y carpinteros utilizan en su trabajo.

iii. No es necesario que una persona sepa leer ni escribir, para que pueda realizar cálculos mentales como sumas y restas.

iv. Los incas en Sudamérica, los mayas en Centroamérica, los yoruba en África y los árabes en Irak crearon, cada uno, sus propios sistemas de numeración. De esta misma manera, cualquier grupo cultural en el mundo puede desarrollar su propio sistema de numeración.

v. Las matemáticas, el lenguaje, la música etc., forman parte de la cultura.

vi. Las matemáticas son creadas por el hombre y responde a las necesidades particulares de una sociedad a lo largo de su historia.

vii. Las tribus africanas carecen de nociones matemáticas.

viii. Fuera de la escuela no se aprenden matemáticas.

ix. Hay comunidades indígenas en la Amazonía brasilera que tienen palabras en su lengua local para contar solo hasta tres, después dicen muchos. Esto se debe a falta de educación escolar.

x. Existen comunidades que relacionan el número cinco con la mano, entonces para decir diez, dicen dos manos. Esta es una forma muy precaria de simbolizar los números.

xi. Para formar estudiantes críticos es importante que los alumnos dominen la estadística.

xii. Si mis compañeros de clase de matemáticas me dicen que soy bueno en esta materia, esto influye en mi rendimiento académico.

xiii. No importa ser niña o niño para ser bueno en matemáticas.

xiv. Las creencias y sentimientos hacia las matemáticas afectan su aprendizaje.

xv. El aprendizaje de las matemáticas se debe sólo a una buena metodología utilizada por el profesor.

xvi. Las matemáticas y las ciencias sociales no se relacionan.

xvii. Las matemáticas son una herramienta útil para entender los problemas sociales actuales.

Figura 2 - Instrumento utilizado en la entrevista grupal, constituido por diecisiete frases. 
Otro registro producido fue a partir del diario de campo que posibilitó detallar el contexto, la titulación de los maestros, registrar la organización y el desarrollo del curso.

\subsection{Descripción del caso: Curso de Formación}

\subsubsection{Contexto}

El caso: Curso de Formación de Maestros desde la Etnomatemática -CFME se llevó a cabo en el municipio de Tumaco, ubicado en el Departamento de Nariño, en la zona sur occidental de Colombia. El municipio tiene una población de 145.344 personas, de las cuales el $95 \%$ son afrodescendientes, $3 \%$ indígenas y $2 \%$ mestizos. Debido a su alto porcentaje de población afrocolombiana, Tumaco fue declarado un municipio etnoeducador, lo que significa que pueden integrar las prácticas culturales de la comunidad en los currículos. Esto está reglamentado, en Colombia, por medio de la Ley General de Educación, 115 de 1994, en su capítulo 3: Educación para grupos étnicos.

\subsubsection{Historia y justificación del Curso}

El CFME fue motivado, por un lado, por el interés de los maestros de incorporar la etnomatemática al aula de clase. Los maestros reconocen la importancia de recuperar los saberes matemáticos autóctonos que, en muchos casos, se han perdido o se encuentran inmersos en la práctica cotidiana (en los ritos, en la agricultura, en las mochilas etc.), y ven en la etnomatemática un camino para dar mayor significado al aprendizaje de las matemáticas. Por el otro, el CFME fue motivado por las carencias detectadas en educación en la comunidad de Tumaco y que se presentan en el documento marco para la construcción del plan decenal de educación "Planes de vida para comunidades ancestrales: hacia un plan decenal de educación para el municipio de Tumaco 2011-2021” de Jaramillo et al. (2011).

\subsubsection{Estructura del Curso}

El Curso se organizó en tres fases: Planeación, Implementación y Resultados. La Planeación contenía la etapa Diseño cooperativo del curso (5 horas). En esta etapa se realizaron dos reuniones con los maestros para definir las características del curso: objetivos, contenidos, la población beneficiaria, tiempos etc.; la Implementación tenía tres etapas: la 
primera etapa: Teórica-Conceptual (32 horas) se dividió en tres momentos: a. Concepciones de los maestros sobre las matemáticas, b. Relación de la cultura y el currículo y c. Investigación de matemáticas extraescolares en prácticas culturales de la comunidad. La segunda etapa: Diseño de actividades (32 horas), la Tercera etapa: aplicación (40 horas); Los Resultados contenía la etapa de Evaluación del curso por parte de los maestros participantes (2 horas).

El Cuadro 2 expone las fases, etapas, momentos y la forma de trabajo utilizada con los maestros.

\begin{tabular}{|c|c|c|c|}
\hline Fase & Etapa & Momentos & Forma de trabajo \\
\hline \multirow[t]{2}{*}{ Planeación } & \multirow[t]{2}{*}{$\begin{array}{l}\text { Diseño } \\
\text { cooperativo del } \\
\text { curso }\end{array}$} & $\begin{array}{l}\text { Reunión para elaborar un pre-diseño del } \\
\text { curso y definir: objetivos, contenidos, } \\
\text { fases, maestros a quien iba orientado el } \\
\text { curso, duración, horarios, lugar }\end{array}$ & Discusión grupal \\
\hline & & Reunión para socializar el diseño del curso & Discusión grupal \\
\hline \multirow[t]{5}{*}{ Implementación } & \multirow[t]{3}{*}{$\begin{array}{l}\text { Teórica- } \\
\text { Conceptual }\end{array}$} & $\begin{array}{l}\text { Concepciones de los maestros sobre las } \\
\text { matemáticas }\end{array}$ & Discusión grupal \\
\hline & & Relación de la cultura y el currículo & $\begin{array}{lr}\text { Lectura } & \text { de } \\
\text { documentos, trabajo en } \\
\text { grupos y discusión } \\
\text { grupal }\end{array}$ \\
\hline & & $\begin{array}{l}\text { Investigación } \quad \text { de } \quad \text { matemáticas } \\
\text { extraescolares en prácticas culturales de la } \\
\text { comunidad }\end{array}$ & $\begin{array}{l}\text { Trabajo de } \\
\text { investigación por } \\
\text { grupos y exposición de } \\
\text { los resultados }\end{array}$ \\
\hline & $\begin{array}{ll}\text { Diseño } & \text { de } \\
\text { actividades }\end{array}$ & Diseño de las actividades & \multirow[t]{2}{*}{$\begin{array}{l}\text { Metodología } \\
\text { de }^{\text {clase }}{ }^{2}\end{array}$} \\
\hline & Aplicación & $\begin{array}{lccc}\text { Puesta en juego de las actividades } \\
\text { diseñadas } & y & \text { Autoevaluación } & \text { y } \\
\text { coevaluación del trabajo en clase }\end{array}$ & \\
\hline Resultados & Evaluación & $\begin{array}{l}\text { Evaluación del curso por parte de los } \\
\text { maestros participantes }\end{array}$ & $\begin{array}{l}\text { Reflexión individual } \\
\text { por escrito }\end{array}$ \\
\hline
\end{tabular}

Cuadro 2 - Fases, etapas, momentos y forma de trabajo del Curso de Formación desde la Etnomatemática.

En este artículo sólo nos referimos al momento Concepciones de los maestros sobre las matemáticas, que se relaciona con las conjeturas 1 y 2 ya planteadas.

\subsubsection{Participantes en el Curso}

El grupo que participó en el CFME fue de 28 maestros: 23 de ellos laboran en la educación básica primaria, y 5 en la educación básica secundaria del municipio de Tumaco. Todos tienen a cargo el área de matemáticas y varios de ellos trabajan en zonas rurales del

\footnotetext{
${ }^{2}$ Esta metodología busca por parte de los maestros una cualificación permanente, un trabajo reflexivo y crítico sobre su práctica y consta de cuatro etapas: 1. La planeación en grupo de las actividades, 2 . La implementación de la actividad y observación de clase, 3. La auto-evaluación y la co-evaluación, y 4. El rediseño de las actividades (HART; ALSTON; MURATA, 2011)
} 
municipio. Su formación profesional es muy diversa, lo cual enriqueció las discusiones y las perspectivas frente a la enseñanza y el aprendizaje de las matemáticas. Tales profesiones son: Licenciatura en ciencias sociales, Licenciatura en básica primaria con énfasis en informática, en lengua castellana, en ciencias naturales y medio ambiente, Normalistas, Licenciatura en comercio y contaduría, Licenciatura en matemáticas y Licenciatura en sicología de familia. Finalmente, es importante señalar que los maestros se inscribieron al curso de formación de manera voluntaria.

\subsubsection{Descripción del momento del curso: concepciones de los maestros sobre las matemáticas}

Al iniciar la fase de implementación, primera clase del curso, se presentaron los objetivos generales, que en la fase de planeación se habían concertado con los maestros, se precisaron los horarios de trabajo en común acuerdo y se dio inicio al momento Concepciones de los maestros sobre las matemáticas. En una mesa redonda, el profesor-investigador presentó a los maestros un instrumento constituido por una lista de 17 frases, con la intención de que los maestros fueran expresando con su propio lenguaje sus concepciones de las matemáticas y su aprendizaje al dar sus opiniones, en favor o en contra, de lo afirmado en cada frase y discutieran sobre éstas de manera organizada. No se entregó ninguna lectura previa. El instrumento fue proyectado frase por frase.

\section{Análisis de datos y resultados}

\subsection{Primer nivel de análisis y resultados}

El material empírico analizado fueron las grabaciones de audio de la sesión de entrevista grupal. Esta entrevista fue transcrita para realizar un análisis de su contenido. Análisis que implicó un proceso de codificación abierta y de comparación constante (STRAUSS; CORBIN, 2002) de las unidades de información correspondientes a las argumentaciones de los maestros. El proceso de codificación y análisis se apoyó en el uso del software Atlas.ti, versión 6.2. Las categorías que emergieron del análisis de dichas unidades de información, su descripción y la componente (relativa a las conjeturas 1 y 2) a la que pertenecen se presentan en el Cuadro 3. 


\begin{tabular}{|c|c|c|}
\hline $\begin{array}{l}\text { Categorías } \\
\text { emergentes }\end{array}$ & Descripción & Componente \\
\hline $\begin{array}{l}\text { Matemática } \\
\text { escolar }\end{array}$ & $\begin{array}{l}\text { Se refiere a los contenidos matemáticos enseñados en el aula de clase, que } \\
\text { son caracterizados por un nominalismo formal de los conceptos } \\
\text { matemáticos }\end{array}$ & \multirow{2}{*}{$\begin{array}{l}\text { Concepciones } \\
\text { de los maestros } \\
\text { sobre las } \\
\text { matemáticas }\end{array}$} \\
\hline $\begin{array}{l}\text { Matemática } \\
\text { extraescolar }\end{array}$ & $\begin{array}{l}\text { Se refiere al reconocimiento de prácticas matemáticas fuera del aula de } \\
\text { clase, haciendo uso de un nominalismo popular para referirse a conceptos } \\
\text { o prácticas matemáticas que se realizan en gremios como pescadores, } \\
\text { tejedoras, comerciantes, artesanos, agricultores etc. }\end{array}$ & \\
\hline $\begin{array}{l}\text { Aprendizaje } \\
\text { situado }\end{array}$ & $\begin{array}{l}\text { Se refiere al aprendizaje que se realiza apoyándose en actividades } \\
\text { contextualizadas que incorporan prácticas cotidianas y que permiten que } \\
\text { los estudiantes adquieran mayor significado de los conceptos. }\end{array}$ & \multirow{5}{*}{$\begin{array}{l}\text { Matemáticas } \\
\text { extraescolares y } \\
\text { su relación con } \\
\text { el currículo }\end{array}$} \\
\hline $\begin{array}{l}\text { Integración } \\
\text { curricular }\end{array}$ & $\begin{array}{l}\text { Hace referencia a la posibilidad de integrar elementos de la cultura y la } \\
\text { comunidad en el currículo escolar. }\end{array}$ & \\
\hline Evaluación & Se refiere a la aplicación de evaluaciones estandarizadas nacionales. & \\
\hline $\begin{array}{l}\text { Interés en } \\
\text { cambios } \\
\text { curriculares }\end{array}$ & $\begin{array}{l}\text { Hace referencia al interés positivo o negativo de cada uno de los agentes } \\
\text { educativos, como maestros, directivos docentes y administradores } \\
\text { educativos }\end{array}$ & \\
\hline $\begin{array}{l}\text { Relaciones } \\
\text { intergeneracio } \\
\text { nales }\end{array}$ & $\begin{array}{l}\text { Informa sobre las relaciones entre jóvenes y adultos mayores realizando } \\
\text { una tarea matemática }\end{array}$ & \\
\hline
\end{tabular}

Cuadro 3 - Análisis de las unidades de información: componentes, categorías emergentes y su descripción.

Estas categorías emergentes representan ya un primer resultado del análisis de los datos.

\subsection{Segundo nivel de análisis y resultados}

A continuación los datos fueron analizados a la luz de los constructos de la filosofía de Wittgenstein, para aclarar la relación causa efecto entre nuestras dos conjeturas. Con este segundo nivel de análisis tratamos de obtener una interpretación alusiva a las relaciones entre las categorías emergentes, del primer nivel de interpretación, referidas a la conjetura 1 y la conjetura 2.

\subsubsection{Resultados sobre las concepciones de los maestros con relación a las matemáticas}

Mediante el análisis de la información logramos identificar al menos dos categorías, que corresponden a las dos concepciones de los maestros acerca de las matemáticas. La primera tiene que ver con la caracterización de las matemáticas escolares mediante el formalismo y los sistemas de representación estandarizados. La segunda tiene que ver con el reconocimiento de matemáticas extraescolares, caracterizadas por sistemas de representación locales y reglas arbitrarias de acuerdo con el contexto. 
Estas dos categorías emergentes se analizaron con las herramientas filosóficas que nos brinda Wittgenstein: Juegos de lenguaje, formas de vida y semejanzas de familia.

Un resultado interesante de dicho análisis fueron las reflexiones presentadas por el maestro A (cuadro 4) sobre la frase: Las mujeres africanas que tejen figuras como cuadrados, círculos, triángulos, etc. en los cestos o en la ropa tienen nociones de geometría.

\begin{tabular}{|ll|}
\hline Maestro A: & Yo pienso que ellas no tienen nociones de geometría porque ellas la palabra \\
& ni la conocen, geometría, de pronto la figura de una forma empírica la \\
& pueden elaborar pero no saben ni siquiera las medidas, o sea, no saben cómo \\
& se elabora de una forma científica sino, de pronto de una forma empírica la \\
& han aprendido a hacer.
\end{tabular}

Cuadro 4 - Reflexiones del maestro A.

En palabras de Wittgenstein (1996) puede interpretarse en el discurso del maestro A el reconocimiento de dos juegos de lenguaje diferentes. Por un lado, los juegos de lenguaje relacionados con la forma de vida de las tejedoras, que carece de patrones de medida y desconocen el vocabulario matemático especializado para referirse a las figuras que diseñan en sus tejidos (matemáticas extraescolares). Por el otro, están los juegos de lenguaje que habitan en las formas de vida escolares, donde existe la necesidad imperativa de conocer un vocabulario especializado, los patrones de medida estandarizados y las propiedades de las figuras reconocidas como geométricas (matemáticas escolares). Dicho maestro encuentra semejanzas de familia entre estos dos juegos de lenguaje, pues reconoce que las tejedoras elaboran figuras que son parecidas a las figuras geométricas, pero critica el proceso de elaboración, las reglas de la forma de vida de las tejedoras, que no son las adecuadas desde el punto de vista de las formas de vida escolares. Esto lo interpretamos como una sobrevaloración de los juegos de lenguaje que habitan en formas de vida escolares sobre los demás y una descalificación de las reglas de la forma de vida de las tejedoras.

En este mismo sentido, interpretamos las reflexiones de la maestra B (cuadro 5) que expresó:

\begin{tabular}{|ll|}
\hline Maestra B: & Yo comparto lo que está diciendo el profesor, de pronto eso si es una figura, \\
& pero ni siquiera saben lo que están haciendo, solamente es una figura ancestral \\
& y lo llevan a la práctica, ni siquiera saben ellas qué es lo que están realizando. \\
& Ya cuando llegan a lo que es matemático ya ellas no saben lo que han hecho. \\
Profesor: & ¿Será que en su cultura no tienen una palabra para designar esa figura? \\
Maestra B: & Si, de lo que es la práctica de ellas, prácticamente lo ancestral, lo que ellas \\
& aprendieron en su entorno. Por ejemplo, mujeres que tejen el canasto ellas van \\
& haciendo los ojos de los canastos, con una medida exacta, la altura y el ancho \\
& pero ellos no saben qué están aplicando allí matemáticamente, solamente la \\
& práctica eso las lleva a hacer, inventar, a ser unas verdaderas personas sabias. \\
Profesor: & ¿Qué cosas la llevan a ser unas personas sabias? \\
Maestra B: & Porque en la práctica ellas saben cuántos ojos del canasto utilizan a lo alto para \\
\hline
\end{tabular}


meterle cangrejos y cuántos ojos utilizan para lo ancho, cuántos cangrejos caben en ese canasto, eso les da la oportunidad de ser una persona sabia.

Cuadro 5 - Reflexiones de la maestra B.

En el discurso de la maestra $\mathrm{B}$ es aún más claro el reconocimiento de juegos de lenguaje matemáticos en las prácticas de las tejedoras y que poseer ese conocimiento les hace ser personas sabias. Pero al igual que el Maestro A, estos conocimientos son relegados cuando son evaluados a luz de los juegos de lenguaje propios de las formas de vida escolares.

Veamos, ahora, las reflexiones del Maestro C (cuadro 6) que tiene una postura contraria a las del Maestro A y la Maestra B.

\begin{tabular}{|c|c|}
\hline Maestro C: & $\begin{array}{l}\text { Yo considero que ellas si sabían la geometría, yo creo que en cada etapa de la } \\
\text { vida sabemos utilizar esos términos, porque la geometría es un término } \\
\text { científico, pero en diferentes culturas manejan otros términos de palabras y } \\
\text { siempre lo que se ha cambiado en las diferentes etapas de la vida son los } \\
\text { términos. Nosotros sabemos que nos metieron en los términos estándares, lo que } \\
\text { ha cambiado son los términos, pero sí se ha sabido qué manejo le han dado a ese } \\
\text { concepto. }\end{array}$ \\
\hline
\end{tabular}

Cuadro 6 - Reflexiones del maestro C.

Este maestro, también reconoce las semejanzas de familia existentes entre los juegos de lenguaje de la matemática escolar, a los que él se refiere como científico o los términos estándares y los juegos de lenguaje de las formas de vida de las mujeres tejedoras de África, pero a diferencia de los otros, reconoce que la mujeres tejedoras sí, saben geometría, pero utilizan términos distintos para nombrar los objetos. Es decir, está colocando al mismo nivel epistemológico los juegos de lenguaje extraescolares y los juegos de lenguaje escolares, a diferencia de los maestros A y B que, aunque reconozcan semejanzas de familia entre estos dos juegos de lenguaje, terminan jerarquizando uno sobre el otro.

Estas dos posturas ilustran las dos categorías que constituyen las concepciones sobre la naturaleza de las matemáticas de unos y otros. No hay un consenso entre ellas. Dichas concepciones comparten las características señaladas por Vilela (2007) y que fueron presentadas en el Cuadro 1.

\subsubsection{Resultados sobre las Matemáticas extraescolares y su relación con el currículo}

Al realizar el ejercicio de categorización de los datos, logramos detectar al menos cinco categorías emergentes que corresponden a esta componente y que fueron descritas en el cuadro 3. Estas son: Aprendizaje situado, Integración curricular, Evaluación, Interés en cambios curriculares y Relaciones intergeneracionales. 
En relación con la categoría Aprendizaje situado, un resultado importante se vincula a las reflexiones presentadas por el maestro E (cuadro 7) y las maestras D y B (cuadro 8) sobre la frase: Fuera de la escuela no se aprenden matemáticas.

\begin{tabular}{|ll|}
\hline Maestro & Un señor que yo conocí, sabía que el barco que llegaba, según el tamaño del \\
E: & barco, cuántos centímetros cúbicos de madera iban ahí, y esos tales centímetros \\
& cúbicos que había en el barco, él ya sabía cuántos millones le daban, cuántas \\
& toneladas de madera, y él no sabía leer. Yo mismo me quedaba sorprendido \\
& como lo hacía. Entonces, la gente me decía, no ese señor es un duro en eso, a \\
& través de la práctica hace tiempo maneja eso.
\end{tabular}

Cuadro 7 - Reflexiones del maestro E.

Este maestro E señala un ejemplo de los juegos de lenguaje emergentes en formas de vida individuales, en este caso, en el cálculo del volumen ocupado por la madera y el peso total de ésta. Además de señalar la presencia de un proceso de aprendizaje personal de las matemáticas en contextos no escolares.

Veamos, ahora, la reflexión sobre esta misma frase de dos maestras:

\begin{tabular}{|c|c|}
\hline Maestra D: & $\begin{array}{l}\text { Pero mire que muchas veces nosotros los profesores o padres de familia, } \\
\text { cuando uno saca el niño al tablero, y dice: no, es que ese niño no sabe sumar } \\
\text { ni sabe restar; mire, perdió porque ese niño no sabe sumar ni restar, pero } \\
\text { tenemos en cuenta solo el concepto de acá (escuela), pero cuando uno lo } \\
\text { manda a la tienda con un billete de } \$ 5000 \text { (pesos colombianos), el niño no se } \\
\text { deja robar, el niño sabe sumar, sabe restar y tomamos en cuenta lo de acá y } \\
\text { no lo de afuera donde el niño se desenvuelve. A la hora del recreo, cuando va } \\
\text { a comprar a la tienda, dice aquí me falta. }\end{array}$ \\
\hline Maestra B: & $\begin{array}{l}\text { Los niños que van a conchar }{ }^{3} \text { al mar, los niños son expertos contando } \\
\text { cuántas conchas hay y cuentan por pares o de cinco en cinco y eso es una } \\
\text { facilidad con la que cuentan y yo como profesora ya me es difícil hacerlo. }\end{array}$ \\
\hline
\end{tabular}
Cuadro 8 - Reflexiones de las maestras D y B

Estas maestras, reconocen la existencia de juegos de lenguaje en formas de vida distintas a la forma de vida escolar y reflexionan sobre la poca o ninguna atención que a estos juegos del lenguaje se le presta en la escuela y sobre la habilidad de los niños de cálculo mental por fuera del aula. En otras palabras, las Maestras D y B encuentran, en las formas de vida extraescolares de los niños, juegos de lenguaje individuales, significados construidos por ellos, que ellas reconocen como válidos, pero que poco o nada son tenidos en cuenta en el aula de clase, al evaluar o reconocer lo que un niño sabe de matemáticas.

Encontramos, aquí, la imperativa necesidad de tener en cuenta el contexto y las matemáticas extraescolares en los procesos de enseñanza y aprendizaje de las matemáticas. En palabras de Sagástegui (2004, p.33):

[...] el aprendizaje situado exige en la escuela una actividad creativa de interpretación del mundo; requiere que los estudiantes operen en situaciones "reales" y "auténticas" semejando las formas de aprendizaje que se producen en la vida

\footnotetext{
${ }^{3}$ Acción de recoger conchas en la playa.
} 
cotidiana, en donde los sujetos se encuentran inmersos en el marco de sentido de una cultura.

Estas reflexiones señalan una limitación en la escuela actual, puesto que en esta se presenta una ruptura con el entorno cotidiano del estudiante.

Los resultados clasificados bajo la categoría: Integración curricular, se encontró que estaban en consonancia con la tipología presentada por Vilela (2007): Interés Cognitivo, Amplificador y Político.

Integración curricular- interés Cognitivo: veamos la reflexión de la maestra I (cuadro 9) sobre cómo, al integrar las matemáticas extraescolares al aula de clase, éstas ayudan a que el estudiante tenga un mayor significado del contenido matemático escolar.

\begin{tabular}{ll}
\hline Maestra I: & no es que se va a dejar de la lado la matemática como tal, porque eso es lo que \\
& nos van a evaluar, pero se le facilita más para que el muchacho aprenda, yo \\
& tengo varias experiencias [...] (subrayado nuestro).
\end{tabular}

Cuadro 9 - Reflexiones de la maestra I.

Esta maestra deja ver la relación de superioridad que tienen los juegos de lenguaje de formas de vida escolares sobre los juegos de lenguaje de formas de vida extraescolares, y deja claro que éstas (las matemáticas extraescolares) son solo un medio para comprender mejor las matemáticas como tal, y que además serán esas las que el gobierno nacional evaluará. Aunque esta forma de integrar las matemáticas extraescolares al aula de clase pareciera una posibilidad, es, a nuestro juicio, una limitación, en tanto que los juegos de lenguaje de formas de vida extraescolares son vistos como un medio, más parecido a un elemento motivador o una curiosidad, que como un objeto matemático de estudio.

Integración curricular- interés Amplificador: veamos, ahora, una reflexión del maestro E (cuadro 10) sobre acrecentar el conocimiento matemático escolar al incorporar las matemáticas extraescolares al aula y reflexionar con ellos sobre éstas:

\begin{tabular}{|c|c|}
\hline Maestro E: & $\begin{array}{l}\text { [...] creo que la clave está en trabajar con los estudiantes que las medidas de } \\
\text { nuestro terreno, de nuestros ancestros les sirvieron cuando no estaba la medida } \\
\text { estándar, y que en un momento dado, también nosotros, más que todo en las } \\
\text { zonas apartadas, podemos utilizarlas, que si no tenemos el metro no nos } \\
\text { vamos a varar, no son tan precisas pero no nos vamos a varar por eso. Creo } \\
\text { que ahí está la clave, lo que decía la compañera, vaya mida, ¿con qué?, no!, } \\
\text { mida, con pasos, creo que ahí está la clave. }\end{array}$ \\
\hline
\end{tabular}

Cuadro 10 - Reflexiones del maestro E.

Este maestro encuentra semejanzas de familia entre los juegos de lenguaje escolares y los extraescolares, permitiendo que uno se enriquezca del otro, viéndolo como un análogo, como un conocimiento que es posible utilizar para la resolución de un problema matemático 
de la vida cotidiana, en este caso, un problema de medición. Vemos, aquí, una posibilidad de que las matemáticas escolares y las matemáticas extraescolares convivan simbióticamente en el currículo escolar, reconociendo las potencialidades y restricciones de uno y de otro. A este respecto D'Ambrosio menciona, en una entrevista realizada por Blanco-Álvarez (2008, p.23), que el profesor debe dar a los niños:

[...] la palabra y proponer un problema general, no enseñar cómo resolverlo, sino dejar que cada uno haga la solución que tiene a partir de su ambiente cultural, por ejemplo, un problema que sea relativo a espacio, distribución de espacio, contar el tiempo, cómo ustedes hacen eso, y ahí dejar que ellos hablen de su solución al problema que está impregnada de su herencia cultural, y después el maestro debe hacer una comparación entre las varias formas: afrodescendientes, indígenas, mestizos, ... de resolver el problema y el maestro debe entonces presentar su forma de hacerlo, que es la manera académica, entonces el maestro no dice: olvida la tuya, ésta es la correcta, no!. Debe decir: tú haces así, yo hago así y claro en algunas cosas será mejor hacerlo a la manera del profesor, hay otras veces que será mejor hacerlo a su manera.

Integración curricular- interés Político: por último, presentamos una reflexión de la maestra F (cuadro 11) que llama la atención sobre el reconocimiento de las matemáticas extraescolares de la cultura tumaqueña:

\begin{tabular}{|c|c|}
\hline Maestra F: & $\begin{array}{l}\text { Lo importante es llevar mucho a los niños a la práctica, como dijo el profe, uno } \\
\text { hace un ejemplo y se queda en el ejemplo, así como se hace práctica con el } \\
\text { metro y uno los manda a medir, así mismo hay que practicar lo nuestro, ¿si me } \\
\text { entiende?, para darle la importancia de la parte etno (subrayado nuestro). }\end{array}$ \\
\hline
\end{tabular}

Cuadro 11 - Reflexiones de la maestra F.

En esta reflexión se puede notar el reconocimiento, en las distintas formas de vida que conforman la cultura tumaqueña, de juegos de lenguaje propios, autóctonos, y la maestra llama la atención de la importancia de reivindicarlos. La propuesta de esta maestra frente a las matemáticas extraescolares ya no solo tiene un interés amplificador, sino que busca ir más allá, a una acción de inclusión social, no solo de las matemáticas escolares, sino también, de los niños en su cultura. Esta es una postura que permite pensar en posibilidades equitativas y con valor de reivindicación social de una integración de las matemáticas extraescolares, a largo plazo, en el currículo escolar.

Por otra parte, algunas reflexiones de los maestros fueron clasificadas como Evaluación, en tanto se referían a los procesos de evaluación del sistema educativo colombiano. Veamos lo que dice el maestro A y la maestra I (cuadro 12):

\begin{tabular}{|c|c|}
\hline Maestro A: & $\begin{array}{l}\text { Yo pienso que anteriormente la educación es igual que ahora, ellos siempre } \\
\text { nos han medido con resultados y el ICFES }{ }^{4} \text {, los que evalúan la educación en } \\
\text { Colombia nunca le va a mandar el niño a la tienda, lo que lleva es un } \\
\text { formulario que tiene que resolverlo con lapicero y papel, entonces de qué le }\end{array}$ \\
\hline
\end{tabular}

\footnotetext{
${ }^{4}$ Instituto Colombiano para la Evaluación de la Educación ICFES.
} 
sirve saber que usted sabe, si los resultados no le están mostrando que usted sabe, tiene que regirse a lo que la educación como tal está marcando (subrayado nuestro).

Cuadro 12 - Reflexiones del maestro A y la maestra I.

Podemos interpretar que este maestro tiene prevenciones sobre la integración de la matemática extraescolar al currículo, pues siente que dicha integración no es valorada ni tenida en cuenta en las evaluaciones nacionales estandarizadas. También, argumenta que para el sistema educativo son más importantes los resultados que el aprendizaje de los estudiantes.

A este respecto, D’Ambrosio (2011, p. 208) menciona que:

En la educación, viene existiendo el reconocimiento de la importancia de las relaciones interculturales. Pero, lamentablemente, todavía hay una reluctancia en el reconocimiento de las relaciones intraculturales. Todavía, se insiste en colocar niños en grados de acuerdo con la edad, ofrecer el mismo currículo en un mismo grado, llegando al absurdo mayor de evaluar grupos de individuos con test estandarizados. ¡Se trata, efectivamente, de una tentativa de pasteurizar las nuevas generaciones! (Traducción nuestra).

Vemos, en las evaluaciones nacionales estandarizadas, una limitación para realizar, de manera permanente, una integración curricular de las matemáticas extraescolares, puesto que los maestros prefieren presentar los contenidos de las matemáticas escolares que se esperan en los Estándares de competencias básicas en matemáticas y tener buenos resultados en las evaluaciones. Esto ejerce mucha presión en los maestros, porque los bajos resultados en dichas pruebas tienen consecuencias económicas y sociales para la institución educativa y el maestro.

Concordamos con Blanco-Álvarez et al. (2014, p. 254) en la necesidad de "la creación de unas políticas públicas que valoren y legitimen otras formas de construir currículos, que consideren las particularidades e intereses de cada una de las culturas que constituyen el país."

Otras reflexiones de los maestros fueron clasificadas como Interés en cambios curriculares en tanto están relacionadas con los intereses de distintos agentes educativos. Veamos la reflexión del maestro E (cuadro 13) al respecto:

\begin{aligned} \hline Maestro E: & ${[\ldots]$ cualquier innovación, cualquier cosa nueva, creo que nosotros estamos en esa $} \\ &$ tarea de jalarle, yo lo que veo es que no hay esa integridad, a qué me refiero, los \\ & profesores estamos a una lado y somos los que estamos cargando el bulto, muchas \\ & veces los directivos están en otro cuento, muchas veces ni les interesa, estamos \\ & hablando de rectores, coordinadores, secretaria de educación y si vamos hacia arriba \\ & ni digamos [...] (subrayado nuestro). \end{aligned}

Cuadro 13 - Reflexiones del maestro E.

Encontramos, aquí, que en ocasiones los directivos docentes o la administración educativa se presenta como una limitación en tanto que no le interesa la integración de juegos de lenguaje de formas de vida extraescolares al aula. Una posible explicación es que también 
se sientan presionados por las pruebas nacionales o por la dificultad que presenta el control y guía de lo diverso, frente a la simplicidad de lo estandarizado.

Finalmente, otras reflexiones fueron clasificadas como Relaciones intergeneracionales pues corresponden a actividades donde participan padres de familia e hijos. La maestra F (cuadro 14) comenta que:

\begin{tabular}{|ll|}
\hline Maestra F: & (...) así mismo hay que practicar lo nuestro, ¿si me entiende?, para darle \\
& la importancia de la parte etno, porque los estudiantes dicen, al menos en \\
& el caso nuestro, uno los manda allá, una manera bonita en que puede \\
& haber una relación entre el joven y el anciano y la persona de la tercera \\
& edad porque ellos empiezan a preguntar, ah! yo estoy aprendiendo de él y \\
& él está aprendiendo de mí, los señores se sienten importantes y entonces \\
& la relación va a mejorar. En el caso nuestro, allá, se practica mucho y sin \\
& embargo hay muchas contradicciones, colocamos una actividad con el \\
papá o el abuelo a practicar ahí, ustedes dos van a hacer tal cosa y \\
termina el joven diciendo: no!, es que mi papá es más anticuado y por \\
eso no terminamos rápido y el otro le ganó el otro grupo, entonces cómo \\
dejar esa palabra anticuado, lo de ellos es todo lo moderno, lo \\
cibernético, el computador, todas esas cosas (subrayado nuestro).
\end{tabular}

Cuadro 14 - Reflexiones de la maestra F.

Las reflexiones de esta maestra son muy llamativas, en tanto que nos permite tener una mirada distinta, desde otro punto de vista. A lo largo de la investigación y de la interpretación de los datos nos habíamos centrado en las actuaciones de los maestros, clasificándolas como limitaciones o posibilidades, pero aquí vemos que se presenta una limitación que surge de los estudiantes, al calificar los conocimientos de sus abuelos o padres como anticuados y sus conocimientos como modernos. La influencia de los medios de comunicación en esta valoración negativa de lo tradicional es innegable, la psicología social puede explicar el influjo de ciertas valoraciones para producir agrupamientos, por ejemplo por edad o por microcultura tecnológica, que generan sentido de la identidad en niños y jóvenes. Es necesario plantear la cultura ancestral de forma no opuesta a lo moderno o actual, que es lo que se busca por los jóvenes como signo cultural identitario. Esto, sin duda, abre nuevas preguntas y nuevos retos de valorización de la cultura.

\subsubsection{Resultados de nivel global-ecológico influyentes en la formación etnomatemática}

Se propone a los formadores de maestros reflexionar, para organizar la formación, sobre las siguientes emergencias de nuestra investigación, que denominamos categorías de primer orden: a. La postura epistemológica de los maestros acerca de las matemáticas; b. La importancia de considerar el conocimiento situado en el aprendizaje de las matemáticas; c. Los distintos niveles de integración curricular (cognitivo, amplificador y político) de las 
matemáticas extraescolares; d. la influencia de la legislación educativa sobre evaluación en la integración de las matemáticas extraescolares; e. El interés de los directivos docentes en los cambios curriculares, y f. Las relaciones socio-culturales entre los padres de familia y los niños, por su influencia en la aceptación del conocimiento matemático extraescolar. Estos elementos o categorías y las relaciones entre ellas las organizamos en nuevas categorías de orden superior, y no totalmente disjuntas, expresadas en el Cuadro 15.

\begin{tabular}{|c|l|}
\hline Categorías de primer orden & \multicolumn{1}{c|}{ Categorías de segundo orden } \\
\hline a y b & $\begin{array}{l}\text { X: Interna al aula y relativa a los elementos humanos protagonistas } \\
\text { del aprendizaje y la enseñanza }\end{array}$ \\
\hline c y d & $\begin{array}{l}\text { Y: Interna al aula y relativa a los elementos mediadores como los } \\
\text { recursos, las normas institucionales y el currículum: }\end{array}$ \\
\hline d y e & Z: Externas al aula y relativa al Sistema Educativo \\
\hline f & W: Externa al aula y relativa al Sistema Social \\
\hline
\end{tabular}

Cuadro 15 - Elementos a considerar en un modelo de formación etnomatemática de maestros.

La categoría $X$, es interna al aula y relativa a los elementos humanos protagonistas del aprendizaje y la enseñanza (estudiantes y maestros), está constituida por la influencia de la postura epistemológica de los maestros acerca de las matemáticas (a), la importancia de considerar el conocimiento situado en el aprendizaje de las matemáticas (b), y las relaciones entre ambas categorías primarias.

La categoría $\mathrm{Y}$ es interna al aula y relativa a los elementos mediadores como los recursos (c), las normas institucionales, el currículum (d) y las relaciones entre estas dos categorías.

La categoría $\mathrm{Z}$ es externa al aula y compuesta por propuestas relativas al Sistema Educativo: legislación educativa sobre evaluación (d), interés de los directivos docentes en la integración de las matemáticas extraescolares (e), y las relaciones entre ambas.

La categoría W es externa al aula y relativa al Sistema Social (f) y sus diversas variantes (aceptación o rechazo de la cultura ancestral por la microcultura juvenil con sus consecuencias), importantes por su trascendencia en la educación. Las categorías de segundo orden profundizan en los complejos procesos a tener en cuenta al diseñar la etno-formación.

\section{Conclusiones}

Los resultados obtenidos nos permitieron observar las posturas epistemológicas sobre la naturaleza de las matemáticas, permitiéndonos advertir, en varios maestros, una postura de superioridad cuando se habla desde las matemáticas escolares con relación a las matemáticas extraescolares, pero también a señalar posturas distintas, donde las matemáticas extraescolares están al mismo nivel epistemológico que las matemáticas escolares. 
Esto nos lleva a concluir que la Conjetura 1: Los maestros no reconocen prácticas extraescolares como conocimientos matemáticos no es verdadera, puesto que encontramos varios maestros que sí, reconocen semejanzas de familia entre las matemáticas escolares y las matemáticas extraescolares.

Por otro lado, la conjetura 2: Esta convicción obstaculiza el uso de prácticas extraescolares en el aula de clase, también es falsa, en tanto que algunos de los maestros que no reconocen semejanzas de familia entre las matemáticas escolares y las extraescolares; sin embargo, incorporan en sus clases prácticas extraescolares, al menos buscando un interés cognitivo.

Lo que nos llevó a plantear una tipología de maestros frente al uso de elementos culturales en el aula, Cuadro 16:

\begin{tabular}{|l|l|}
\hline \multicolumn{1}{|c|}{ Tipo de maestro } & \multicolumn{1}{c|}{ Descripción } \\
\hline Formalista. & $\begin{array}{l}\text { Trabaja, en el aula, solo matemáticas escolares, ya que no considera } \\
\text { conocimiento las matemáticas extraescolares. }\end{array}$ \\
\hline $\begin{array}{l}\text { Falso } \\
\text { etnomatemático }\end{array}$ & $\begin{array}{l}\text { Incluye, en el aula, matemáticas extraescolares por otras razones diferentes } \\
\text { a pensar que son matemáticas, por ejemplo elemento motivador o curioso. }\end{array}$ \\
\hline $\begin{array}{l}\text { Cuasi- } \\
\text { etnomatemático }\end{array}$ & $\begin{array}{l}\text { Aunque reconoce la existencia y la importancia de las matemáticas } \\
\text { extraescolares, no las incluye en el aula, por diferentes razones: falta de } \\
\text { materiales, currículos no flexibles, presión de los directivos etc. }\end{array}$ \\
\hline Etnomatemático & $\begin{array}{l}\text { Trabaja, en el aula, las matemáticas escolares y las matemáticas } \\
\text { extraescolares, reconociendo la importancia y el papel formador de ambas. }\end{array}$ \\
\hline
\end{tabular}

Cuadro 16 - Tipología de maestros.

Además, interpretamos en las reflexiones de los maestros que existen al menos cinco limitaciones que podemos caracterizar como cognitivas, curriculares y socio-culturales, y dos posibilidades curriculares y socio-políticas para la integración de las matemáticas extraescolares en el aula. Estas limitaciones y posibilidades, son los principales factores que mediatizan la conexión entre las creencias epistemológicas y la acción en el aula, también son elementos que responden, parcialmente, al objetivo general de nuestra investigación doctoral.

Así pues, en un curso de formación de maestros orientado desde la etnomatemática, se hace necesario considerar la inclusión de los elementos obtenidos en esta investigación: tipologías de maestros y categorías derivadas de los procesos de aula, como mediadores de las relaciones entre la matemática escolar, las matemáticas extraescolares y la práctica en el aula.

Consideramos que estos hallazgos nos proporcionan un primer nivel de elementos a tener en cuenta a la hora de diseñar cursos de formación para maestros desde una perspectiva etnomatemática.

\section{Agradecimientos}


Agradecemos a todas las maestras y los maestros que nos compartieron su experiencia docente, a la Universidad de Nariño por su activa participación, y a Save the Children International por haber gestionado y financiado este curso de formación.

\section{Referencias}

BLANCO-ÁLVAREZ, H. Entrevista al profesor Ubiratan D'Ambrosio. Revista Latinoamericana de Etnomatemática, San Juan de Pasto, v. 1, n. 1, p. 21-25, feb. 2008.

BLANCO-ÁLVAREZ, H. La postura sociocultural de la educación matemática y sus implicaciones en la escuela. Revista Educación y Pedagogía, Medellín, v. 23, n. 59, p. 59-66, ene./abr. 2011.

BLANCO-ÁLVAREZ, H. Estudio de las actitudes hacia una postura sociocultural y política de la Educación Matemática en maestros en formación inicial. REDIMAT - Journal of Research in Mathematics Education, Barcelona, v. 1, n. 1, p. 57-78, feb. 2012.

BLANCO-ÁlVAREZ, H.; HIGUITA RAMÍREZ, C.; OLIVERAS, M. L. Una mirada a la Etnomatemática y la Educación Matemática en Colombia: caminos recorridos. Revista Latinoamericana de Etnomatemática, San Juan de Pasto, v. 7, n. 2, p. 245-269, jun. 2014.

CARRAHER, T.; CARRAHER, D.; SCHLIEMANN A. L. En la vida diez, en la escuela cero. México: Siglo veintiuno, 2002.

COHEN, L.; MANION, L. Métodos de investigación educativa. Madrid: la Muralla, 1990.

D'AMBROSIO, U. Ethnomathematics and its place in the history and pedagogy of mathematics. En: POWELL, A.; FRANKENSTEIN, M. (Eds.). Ethnomathematics: Challenging Eurocentrism in Mathematics Education. Albany, EE.UU: SUNY Press, 1997. p. 13-24.

D'AMBROSIO, U. A busca da paz como responsabilidade dos matemáticos. Cuadernos de investigación y formación en educación matemática, San José, v. 6, n. 7, p. 201-215, jun. 2011.

D'AMBROSIO, U. The program ethnomathematics: theoretical basis and the dynamics of cultural encounters. Revue Cosmopolis, Ghent n. 3-4, p. 13-41, 2012.

FERREIRA, E. Etnomatemática. Uma proposta metodológica. Rio de Janeiro: Universidade Santa Úrsula, 1997

HART, L. C.; ALSTON, A.; MURATA, A. (EDS.). Lesson study Research and Practice in Mathematics Education: Learning together. New York: Springer, 2011.

JARAMILLO, D. La educación matemática en una perspectiva sociocultural: tensiones, utopías, futuros posibles. Revista Educación y Pedagogía, Medellín, v. 23, n. 59, p. 13-36, ene./abr. 2011.

JARAMILLO, J. B.; JURADO VALENCIA, F.; COLLAZOS, J. (Coord.) Planes de vida para comunidades ancestrales: hacia un plan decenal en educación para el municipio de Tumaco 20112021. Bogotá: Universidad Nacional de Colombia, Facultad de Ciencias Humanas, Instituto de Investigación en Educación, y Alcaldía de Tumaco, Secretaría de Educación, 2011.

KNIJNIK, G. Exclusão e Resistência: Educação Matemática e Legitimidade cultural. Porto Alegre: Artes Médicas, 1996. 
KNIJNIK, G. Diversidad cultural, matemáticas y exclusión: oralidad y escrita en la educación matemática campesina del sur del Brasil. En: GIMÉNEZ, J.; DÍEZ-PALOMAR, J.; CIVIL, M. (Coord.) Educación matemática y exclusión. Barcelona: Editorial Graó, 2007, p. 66-83.

KNIJNIK, G. Differentially positioned language games: ethnomathematics from a philosophical perspective. Educational Studies in Mathematics, New York, v. 80, n. 1-2, p. 87-100, mar. 2012.

KNIJNIK, G.; DUARTE, C. G. Entrelaçamentos e Dispersões de Enunciados no Discurso da Educação Matemática Escolar: um Estudo sobre a Importância de Trazer a "Realidade" do Aluno para as Aulas de Matemática. BOLEMA: Boletim de Educação Matemática, v. 23, n. 37, p. 863-886, 2010.

KNIJNIK, G.; MEREGALLI, J. Educação matemática em cursos de pedagogia: um estudo com professores dos anos iniciais do ensino fundamental. Revista Latinoamericana de Etnomatemática, San Juan de Pasto, v. 5, n. 2, p. 4-20, ago. 2012.

LIZCANO, E. Metáforas que nos piensan: sobre ciencia, democracia y otras poderosas ficciones. Madrid: Ediciones Bajo Cero. 2006.

MONTEIRO, A.; MENDES, J. Prácticas sociales y organización curricular: cuestiones y desafios. Revista Educación y Pedagogía, Medellín, v. 23, n. 59, p. 37-46, ene./abr. 2011

OLIVERAS, M. L. Etnomatemáticas. Formación de profesores e innovación curricular. Granada: Comares, 1996.

OLIVERAS, M. L. Etnomatemáticas. De la multiculturalidad al mestizaje. En: GIMÉNEZ, J.; GOÑI J. M.; GUERRERO S. (Coord.) Matemáticas e interculturalidad. Barcelona: Graó, p. 117-149, 2006.

SAGÁSTEGUI, D. Una apuesta por la cultura: el aprendizaje situado. Revista Electrónica Sinéctica, Jalisco, v. 24, p. 30-39, feb./jul. 2004.

SANTILLÁN, A.; ZACHMAN, P. Una experiencia de capacitación en Etnomatemática. Revista Latinoamericana de Etnomatemática, San Juan de Pasto, v. 2, n. 1, p. 27-42, ene./jul. 2009.

SCHLIEMANN. A. L. La comprensión del análisis combinatorio: desarrollo, aprendizaje escolar y experiencia diaria. En: CARRAHER, T.; CARRAHER, D.; SCHLIEMANN, A. En la vida diez, en la escuela cero. México: Siglo veintiuno editores, 2002. p. 90-105.

STRAUSS, A.; CORBIN, J. Bases de la investigación cualitativa. Técnicas y procedimientos para desarrollar la teoría fundamentada. Medellin, Colombia: Editorial Universidad de Antioquia, 2002.

THOMPSON, A. Teacher's Beliefs and Conceptions: A Synthesis of the Research. En: GROUWS, D. (Ed.) Handbook of Research on Mathematics Teaching and Learning. New York: MacMillan Publishing Company, 1992. p. 127-146.

VILELA, D. Reflexão filosófica acerca dos significados matemáticos nos contextos da escola e da rua. In: SIPEM - SEMINÁRIO INTERNACIONAL DE PESQUISAS EM EDUCAÇÃO MATEMÁTICA, 3., 2006. Águas de Lindóia. Anais... Águas de Lindóia:SIPEM, 2006. CD-ROM.

VILELA, D. S. Matemática nos usos e jogos de linguagem: Ampliando concepções na Educação Matemática. 2007. 247 f. Tesis (Doctorado en Educación: Educación Matemática) Facultad de Educación, Unicamp, Campinas, Brasil, 2007. 
VILELA, D. S. Discussing a philosophical background for the ethnomathematical program.

Educational Studies in Mathematics, v. 75, n. 3, p. 345-358, 2010.

WITTGENSTEIN, L. Investigaciones filosóficas. Barcelona: Ediciones Altaya, 1999.

Submetido em Outubro de 2014. Aprovado em Abril de 2015. 